\title{
Cytokine profile and nitric oxide levels in sera from patients with brucellosis
}

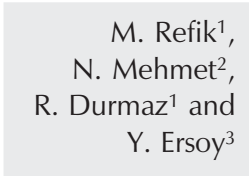

\section{Correspondence}

M. Refik

Department of Microbiology and

Clinical Microbiology

Faculty of Medicine

Inonu University

44069 Malatya

Turkey

E-mail: mrtmehmet@yahoo.com

Received August 20, 2003

Accepted July 28, 2004

\author{
Departments of ${ }^{1}$ Microbiology and Clinical Microbiology, \\ ${ }^{2}$ Biochemistry and Clinical Biochemistry, and \\ ${ }^{3}$ Infectious Diseases and Clinical Microbiology, \\ Faculty of Medicine, Inonu University, Malatya, Turkey
}

\begin{abstract}
The aims of this study were to investigate the serum levels of some cytokines [tumor necrosis factor- $\alpha$ (TNF- $\alpha$ ), interleukin $1 \beta$ (IL-1ß), IL-2R, IL-6, and IL-8] and nitric oxide (NO) levels in patients with untreated brucellosis and to test the correlation of these parameters with each other. The study was conducted on 67 subjects, 37 patients with brucellosis and 30 healthy individuals with no history of Brucella infection. Brucellosis was identified by a positive blood culture and/or increased Brucella antibodies in serological tests in addition to compatible clinical symptoms. Cytokine profile analysis was performed by the immulite chemiluminescent enzyme immunometric assay whose inter- and intra-assay coefficients of variance were 2.6-3.6 and 4.4$8.5 \%$, respectively. The levels of nitrites/nitrates, which are representative of NO levels, were measured by the Griess method. Patients with brucellosis had significantly elevated serum levels of nitrites/ nitrates, IL-2R, IL-6 and IL-8 (mean \pm SD, $102.8 \pm 23.8 \mu \mathrm{mol} / 1,806.1$ $\pm 58.5 \mathrm{U} / \mathrm{ml}, 21.1 \pm 2.3 \mathrm{pg} / \mathrm{ml}$, and $8.8 \pm 1.6 \mathrm{pg} / \mathrm{ml}$, respectively) compared to healthy controls, whereas TNF- $\alpha$ and IL-1 $\beta$ levels were unchanged. No statistically significant correlation was detected between any of the studied cytokine levels and nitrate/nitrite concentrations according to Pearson's linear correlation test. We conclude that only IL-6, IL-8 and IL-2R are elevated in brucellosis and the extent of elevation depends on the severity and clinical pattern of the disease. Moderate elevation in serum NO was comparable to that observed in previous studies. This explains the absence or very rare occurrence of septic shock in brucellosis.
\end{abstract}

Brucellosis is endemic in many Middle East countries including Turkey, where it undermines animal and human health. Brucella spp invade and proliferate within reticuloendothelial cells and cause acute, subacute and chronic disease. The acute form is characterized by weakness, headache, ar-
Key words

- Human brucellosis

- Cytokines

- Nitric oxide

- Interleukin-2

- Interleukin-6

- Interleukin-8 thralgia, malaise, sweating, irregular fever, lower back pain, splenomegaly, and hepatomegaly. In the subacute form, the clinical pattern is more protean, with milder clinical symptoms, with the frequent presence of arthritis and an infrequent occurrence of hepatomegaly. In the chronic form fever is rare 
and focal suppurative lesions in bones, joints, liver, spleen, and eyes may be observed (1).

The immune response against Brucella involves both humoral (Th2) and cell-mediated (Th1) immunity. The Th1/Th2 cytokine ratio seems to be involved in the susceptibility or resistance to Brucella infection. Th1 cytokines confer resistance (2), whereas Th2 cytokines predispose to brucellosis $(3,4)$. Some cytokines can regulate the immune system and play key roles on the outcome of brucellosis. Several studies have shown that $\mathrm{T}$ cell proliferation and Th1 cytokine production are reduced in brucellosis $(5,6)$. Interferon-gamma (IFN- $\gamma$ ) is a well-studied cytokine $(2,5,7,8)$. Other cytokines such as interleukin-2 (IL-2) (5), IL-3, IL-4, IL-8, and IL-12 $(4,9)$, and IL-6 (10) have also been studied in human brucellosis. However, the precise role of other cytokines in human brucellosis has not been thoroughly explored (7). Nitric oxide (NO) is an important bioregulatory molecule. It is labile (half-life $<15 \mathrm{~s}$ ) and is rapidly metabolized to nitrate and nitrite in the presence of oxygen. Although small amounts of NO are constitutively released by endothelial and neuronal nitric oxide enzymes, inducible NO, located mainly in immune cells such as macrophages, is induced and produced in considerable amounts after exposure to potent cytokines $[$ IFN- $\gamma$, tumor necrosis factor- $\alpha$ (TNF- $\alpha$ ) and IL-1] alone or in combination with microbial lipopolysaccharides (LPS) (11). Inducible NO has an antimicrobial activity similar to that of reactive oxygen species and plays a vital role in host defense and immunity, including the modulation of inflammatory responses (2). Although NO has been investigated in animal models $(7,8,12)$, according to our knowledge and Medline survey the role of NO in human Brucella infection has not been evaluated yet. In the present study, we searched for variations in the levels of three inflammatory cytokines, (IL-1ß, TNF$\alpha$, and IL-6), a chemokine, (IL-8), and a receptor of regulatory cytokine IL-2 (IL-2R) in brucellosis patients with an inflammatory reaction and determined their possible correlation with each other and with NO.

The study was conducted on 67 subjects diagnosed and followed at the Turgut Ozal Medical Center, Malatya, Turkey, and comprised 37 brucellosis patients (16 males and 21 females) and 30 controls. Brucellosis was diagnosed on the basis of clinical, bacteriological and serological data. Nine of the patients had acute brucellosis defined by the presence of typical signs and symptoms, e.g., fever, sweating, malaise, back pain, hepatomegaly, myalgia, and arthralgia. Twentyeight patients had the subacute form of the disease, with milder symptoms and arthritis. Control subjects were 30 healthy individuals (14 males and 16 females) with no history of previous Brucella infection. The mean age of patients and controls was $34.7 \pm 9.9$ and $33.7 \pm 6.2$ years, respectively.

The study was carried out in compliance with the guidelines of the Institutional Health Committee of the School of Medicine, Inonu University. All patients and controls signed an informed consent form.

The blood samples collected from untreated patients were inoculated into Bactec Blood culture bottles and incubated in the Bactec 9120 System (Becton Dickinson, Sparks, MD, USA) until a positive signal was observed. Subcultures were obtained on sheep blood agar, chocolate agar and eosin methylene blue (EMB) agar and incubated at $35^{\circ} \mathrm{C}$ in a $5 \% \mathrm{CO}_{2}$ atmosphere for at least 48 $\mathrm{h}$. Small and whitish colonies appeared only on blood and chocolate agar after $48 \mathrm{~h}$ and no growth was observed on EMB agar. The colonies that yielded Gram-negative coccobacilli and were positive for the oxidase and urease tests were identified as Brucella spp. Patient sera were screened by Rose-Bengal slide agglutination and standard tube agglutination (STA) tests.

As soon as brucellosis was identified, blood samples of about $5 \mathrm{ml}$ were collected into sterile tubes without anti-coagulants from 
both groups after an overnight fast. None of the patients or healthy controls was receiving any topical or systemic medication on admission. Following centrifugation of the blood samples at $2000 \mathrm{rpm}$ for $10 \mathrm{~min}$, sera were separated and kept at $-70^{\circ} \mathrm{C}$ until the analysis of cytokines and the NO metabolite.

Cytokine analysis was performed using the immulite chemiluminescent enzyme immunometric assay DPC (Diagnostic Products, Los Angeles, CA, USA). The technique was based on a solid-phase (bead) two-site chemiluminescent enzyme immunometric assay. For each cytokine calibration, a master curve was constructed using the US National Institute for Biological Standards and Controls Reference Preparation according to manufacturer instructions. Since $\mathrm{NO}$ is unstable and rapidly converted to nitrates and nitrites, it is necessary to determine both nitrite and nitrate concentrations in the samples (13). It is common practice to use enzymatic or chemical reduction to convert all nitrates to nitrites in a sample and then measure total nitrite (13). In the present study, serum samples were mixed with granular cadmium and shaken for $2 \mathrm{~h}$ for chemical reduction of nitrate to nitrite, and total nitrite was then measured by spectrophotometry using the Griess reagent. Serum nitrate concentration was calculated by subtracting direct nitrite from total nitrite. All chemicals and biochemicals used for NO determination were obtained from Sigma (St. Louis, MO, USA).

Means and standard deviations were calculated for each parameter and data were analyzed for normal distribution. The means of variables showing normal distribution were compared by the parametric unpaired $t$-test, whereas parameters whose distribution was not normal were compared by the non-parametric Mann-Whitney U-test. Pearson's linear correlation test was used to assess correlation between parameters. The level of significance was set at $\mathrm{P}<0.05$ in all analyses. All analyses were performed using the SPSS 9.0 software.

All acute brucellosis patients and 25 of the 28 subacute patients yielded positive cultures for Brucella species. All patients had a positive Rose-Bengal slide test and STA test with a titer of $1 / 160$ or more. The serological tests used were negative in the control group.

Cytokines and nitrate/nitrite concentrations are shown in Table 1. Both nitrate and nitrite concentrations were significantly higher in patients with brucellosis than in healthy controls $(\mathrm{P}<0.001)$. There was a positive correlation between total and direct nitrites (correlation coefficient $=0.671, \mathrm{P}<$ $0.05)$. Table 1 also presents the mean levels of TNF- $\alpha$, IL-1ß, IL-2R, IL-6, and IL-8 in

\begin{tabular}{|c|c|c|c|c|}
\hline Parameter & $\begin{array}{l}\text { Patients with } \\
\text { brucellosis }(N=37)\end{array}$ & $\begin{array}{l}\text { Controls } \\
(\mathrm{N}=30)\end{array}$ & $\begin{array}{l}\text { Subacute brucellosis } \\
\qquad(N=28)\end{array}$ & $\begin{array}{l}\text { Acute brucellosis } \\
\qquad(N=9)\end{array}$ \\
\hline TNF- $\alpha(\mathrm{pg} / \mathrm{ml})$ & $6.9 \pm 0.8$ & $6.4 \pm 1.3$ & $6.6 \pm 0.3$ & $6.8 \pm 0.9$ \\
\hline IL-1ß (pg/ml) & $4.6 \pm 0.3$ & $4.5 \pm 0.2$ & $4.6 \pm 0.3$ & $4.7 \pm 0.5$ \\
\hline IL-2R (U/ml) & $806.1 \pm 58.5^{*}$ & $581.5 \pm 47.1$ & $761.1 \pm 37.1$ & $851.2 \pm 36.6^{* *}$ \\
\hline IL-6 (pg/ml) & $21.1 \pm 2.3^{*}$ & $4.6 \pm 0.3$ & $18.7 \pm 1.3$ & $23.8 \pm 1.9 * *$ \\
\hline IL-8 (pg/ml) & $8.8 \pm 1.6^{*}$ & $5.3 \pm 0.4$ & $7.6 \pm 0.5$ & $10.1 \pm 1.4^{* *}$ \\
\hline Direct nitrite $(\mu \mathrm{mol} / \mathrm{l})$ & $35.8 \pm 7.1^{*}$ & $18.9 \pm 3.3$ & $31.9 \pm 4.8$ & $40.9 \pm 6.8^{* *}$ \\
\hline Total nitrite $(\mu \mathrm{mol} / \mathrm{l})$ & $102.8 \pm 23.8^{*}$ & $41.0 \pm 9.4$ & $87.1 \pm 13.6$ & $121.2 \pm 18.1^{* *}$ \\
\hline Nitrate $(\mu \mathrm{mol} / \mathrm{l})$ & $67.0 \pm 16.7^{*}$ & $22.1 \pm 6.1$ & $55.2 \pm 8.8$ & $80.3 \pm 13.3^{* *}$ \\
\hline
\end{tabular}

Data are reported as means \pm SD.

${ }^{*} \mathrm{P}<0.05$ compared to control group (Student $t$-test). ${ }^{*} \mathrm{P}<0.05$ compared to subacute brucellosis (MannWhitney U-test). 
sera from patients with brucellosis and controls. Patients had significantly elevated serum levels of IL-2R, IL-6 and IL-8 compared to healthy controls but levels of TNF- $\alpha$ and IL-1 $\beta$ were not different. Moreover, there was no close relation between cytokine production and nitrite/nitrate levels, except for a poor correlation between IL-2R and direct nitrite (correlation coefficient $=0.471, \mathrm{P}<$ $0.05)$. The mean serum levels of TNF- $\alpha$, IL13 , IL-2R, IL-6, IL-8, and nitrite/nitrate according to severity of disease are compared in Table 1. IL-2R, IL-6, IL-8, and nitrite/ nitrate were significantly higher in sera from patients with acute brucellosis than in sera from patients with subacute brucellosis.

Most studies about the role of cytokines and NO in the immunity and course of brucellosis were performed in animal models and in in vitro macrophage cultures $(7,12,14)$, whereas little information is available for human brucellosis. Protective immunity to Brucella infection was conferred not only by antibodies alone but also by activated macrophages, T cells and their cytokines $(9,14)$. In an animal study, it was thought that protection might depend on timing of the cytokine response rather than on the cytokine level (14). The high titer of specific Brucella antibodies $(\geq 1 / 160)$ detected by STA in our patients clearly indicated B cell activation. However, this response may not be sufficient to eliminate infection and adequate antimicrobial treatment is needed for complete recovery.

In the current study, the significant increase in serum IL-6 and IL-8 levels in brucellosis compared to control indicates the role of these cytokines in the inflammatory response. Since IL-6 plays a major role in inflammation and IL-8 is a chemokine that induces chemotaxis and migration of leukocyte subsets (15), this supports the involvement of IL- 6 and IL- 8 in the process and pathogenesis of brucellosis. The receptor of the regulatory cytokine IL-2 (Th1 cytokine) was also significantly increased, possibly regulating the inflammatory reaction by activation of various immune cells and subsequent release of mediators such as cytokines and NO. In an experimental study on mice, it was reported that Brucella LPS did not cause IL-1 production and only a low level of TNF$\alpha$ production (16). In another similar study, it was demonstrated that vaccination of animals with Brucella abortus strain RB51 did not cause an increase in blood TNF- $\alpha$ levels (17). In agreement with these findings, TNF$\alpha$ and IL- $1 \beta$ levels did not differ between infected individuals and controls in the present study.

Pathological conditions associated with increased release of cytokines and LPS such as inflammation or sepsis can increase NO production as well (18). It is well known that Brucella LPS and lipid A directly induce NO production in rats but at a much lower rate than other bacteria such as Escherichia coli (11). Under biological conditions, NO undergoes a number of reactions which tend to involve the formation of both nitrate and nitrite ions. In the present study, nitrate and nitrite levels were considered to be representative of NO production. There was a significant increase in nitrate/nitrite levels, but not so high as to lead to hypotension and septic shock, which were not observed in any patient. The reason seems to be a low or moderate increase in pro-inflammatory cytokine levels and a consequent moderate elevation of NO $(11,19)$. This finding was in agreement with previous data indicating that septic shock was rarely observed or was even absent in animal or human brucellosis but was seen in E. coli infection due to $\mathrm{NO}$ overproduction $(18,20)$. Moreover, our results showed that there were no significant correlations between production of the cytokines studied and nitrite/nitrate levels. This may indicate that increased IL-6 and IL-8 have no significant effect on NO-producing cells. Since NO-inducing cytokines such as TNF- $\alpha$ and IL- $1 \beta$ were not elevated in our patients and IFN- $\gamma$ was significantly increased 
in acute brucellosis as reported in a previous study (9), the most probable reason for a significant increase in NO level in brucellosis patients in the current study may be the effect of Brucella LPS and IFN- $\gamma$ on immune cells. Note that we did not measure IFN- $\gamma$.

One can conclude from this study that only IL-6, IL-8 and IL-2R are elevated in brucellosis and the extent of elevation depends on the severity and clinical pattern of the disease. Moderate elevations in serum NO levels with the simultaneous increase in some cytokines found in our study may not be sufficient to result in septic shock, as is the case for other bacterial infections such as E. coli.

\section{References}

1. Gotuzzo E \& Carrillo C (1998). Brucella. In: Gorbach SL, Bartlett JG, Blacklow NR (Editors), Infectious Diseases. 2nd edn. W.B. Saunders Company, Philadelphia, PA, USA, 1837-1838.

2. Zhan $Y$ \& Cheers $C$ (1993). Endogenous gamma interferon mediates resistance to Brucella abortus infection. Infection and Immunity, 61: 4899-4901.

3. Fernandes DM \& Baldwin CL (1995). Interleukin-10 downregulates protective immunity to Brucella abortus infection. Infection and Immunity, 63: 1130-1133.

4. Galanakis E, Makis A, Bourantas KL \& Papadopoulou ZL (2002). Interleukin-3 and interleukin-4 in childhood brucellosis. Infection, 30: $33-34$

5. Rodriguez-Zapata M, Salmeron I, Manzano L, Salmeron OJ, Prieto A \& Alvarez-Mon M (1996). Defective interferon-gamma production by T-lymphocytes from patients with acute brucellosis. European Journal of Clinical Investigation, 26: 136-140.

6. Giambartolomei GH, Delpino MV, Cahanovich ME, Wallach JC, Baldi PC, Velikovsky CA \& Fossati CA (2002). Diminished production of $T$ helper 1 cytokines correlates with $T$ cell unresponsiveness to Brucella cytoplasmic proteins in chronic human brucellosis. Journal of Infectious Diseases, 186: 252-259.

7. Jiang X \& Baldwin CL (1993). Effects of cytokines on intracellular growth of Brucella abortus. Infection and Immunity, 61: 124-134.

8. Jones S \& Winter AC (1992). Survival of virulent and attenuated strains of Brucella abortus in normal and gamma interferon-activated murine peritoneal macrophages. Infection and Immunity, 60: 3011-3014.

9. Ahmed K, Al-Matrouk KA, Martinez G, Oishi K, Rotimi VO \& Nagataka T (1999). Increased serum levels of interferon-gamma and interleukin-12 during human brucellosis. American Journal of Tropical Medicine and Hygiene, 61: 425-427.

10. Zhan $Y$, Kelso A \& Cheers C (1993). Cytokine production in the murine response to Brucella infection or immunization with antigenic extracts. Immunology, 80: 458-464.

11. Lincolin L, Hoyle HV \& Burnstock G (1997). Nitric Oxide in Health and Disease. 1st edn. Cambridge University Press, Cambridge, UK, 12-26, 105-113.

12. Iyankan L \& Singh DK (2002). The effect of Brucella abortus on hydrogen peroxide and nitric oxide production by bovine polymorphonuclear cells. Veterinary Research Communications, 26: 93102.

13. Moshage $H$, Kok B, Huizenga JR \& Jansen PL (1995). Nitrite and nitrate determinations in plasma: a critical evaluation. Clinical Chemistry, 41: 892-896.

14. Pasquali P, Adone R, Gasbarre LC, Piștoia C \& Ciuchini F (2001). Mouse cytokine profiles associated with Brucella abortus RB51 vaccination or Brucella abortus 2308 infection. Infection and Immunity, 69: 6541-6548.

15. Feghali CA \& Wright TM (1997). Cytokines in acute and chronic inflammation. Frontiers in Bioscience, 2: d12-26.

16. Zhan Y \& Cheers C (1994). Differential induction of macrophagederived cytokines by live and dead intracellular bacteria in vitro. Infection and Immunity, 63: 720-723.

17. Palmer MV, Elsasser TH \& Cheville NF (1998). Tumor necrosis factor-alpha in pregnant cattle after intravenous or subcutaneous vaccination with Brucella abortus strain RB51. American Journal of Veterinary Research, 59: 153-156.

18. Wang M, Qureshi N, Soeurt N \& Splitter G (2001). High levels of nitric oxide production decrease early but increase late survival of Brucella abortus in macrophages. Microbial Pathogenesis, 31: 221230.

19. Gross A, Spiesser S, Terraza A, Rouot B, Caron E \& Dornand J (1998). Expression and bactericidal activity of nitric oxide synthase in Brucella suis-infected murine macrophages. Infection and Immunity, 66: 1309-1316.

20. Lopez-Urrutia L, Alonso A, Nieto ML, Bayon Y, Orduna A \& Crespo MS (2000). Lipopolysaccharides of Brucella abortus and Brucella melitensis induce nitric oxide synthesis in rat peritoneal macrophages. Infection and Immunity, 68: 1740-1745. 